\title{
Building-up a Double Accredited CRM Production Laboratory in an Industrial Environment: A Description
}

\author{
Jürg Wüthrich* and Michael Weber
}

\begin{abstract}
Over the last three years Sigma-Aldrich has built-up a competence centre for CRM (Certified Reference Material) production in Buchs, Switzerland. The new facility is accredited following both ISO/IEC 17025 and ISO Guide 34. The commercial availability of a broad range of different CRMs is of highest importance with respect to the realisation of metrological concepts in practice. This article describes the process of the production and certification of inorganic CRMs of highest quality. This comprises the comprehensive characterisation of the starting materials, the high precision weighing of the compounds, homogenisation and special packaging of these new CRMs.
\end{abstract}

Keywords: Accreditation · Calibration solution · CRM $\cdot$ Measurement uncertainty $\cdot$ Traceability

\section{A New Competence Centre for CRM Production at Sigma-Aldrich}

Sigma-Aldrich offers a portfolio of more than 8000 different analytical standards and is one of the key players in this field. The standards are available under the two brands Fluka and Supelco, whereby a series of Certified Reference Materials (CRM) have been established over the last few years. In the past most of these CRMs have been developed through cooperation with external partners, most of them being National Metrological Institutes (NMI) or other expert laboratories such as IRMM (EU), BAM (Germany) or EMPA (Switzerland). Hereby Sigma-Aldrich acted either as a distributor whereby the CRMs are offered under the original brand such as $\mathrm{BCR}^{\circledR}$ or $\mathrm{ERM}^{\circledR}$ or its own brand e.g. Trace CERT ${ }^{\circledR}$. Other CRMs have been developed in close cooperation with NMIs whereby the CRMs are produced and bottled by Sigma-Aldrich and the certification was performed by the NMI. Together with

${ }^{\star}$ Correspondence: J. Wüthrich

Research and Development

Sigma-Aldrich Schweiz Production $\mathrm{GmbH}$

Industriestrasse 25

CH-9471 Buchs/SG

Tel.: +41817552325

Fax: + 41817552272

E-mail: juerg.wuethrich@sial.com
BAM and EMPA a series of CRMs for titrimetry (acidimetric, complexometric and redox), spectrometry (single- and multielement) and ion chromatography (anions, cations and multi-ion) have been developed successfully over the last ten years.

As an extension of this cooperative approach for the development of CRMs Sigma-Aldrich decided to build-up an in-house competence centre for the production of CRMs in Buchs, Switzerland. Two major reasons triggered this decision: Firstly the demand for CRMs has increased significantly over the last few years since more and more laboratories are following normative guidelines such as ISO/IEC 17025.[1] Secondly EMPA was one of the key partners for CRM development and decided to terminate all their metrological activities in chemistry in 2005. Consequently Sigma-Aldrich had to find a way to keep alive these CRMs. Therefore Sigma-Aldrich jumped at the opportunity to acquire EMPA's equipment for CRM production including clean room workstations, homogenisation and bottling instrumentation and also a series of high-purity starting materials. In addition to the equipment Sigma-Aldrich also incorporated the metrological know-how by hiring the former head of metrology and the leading scientist for certification from EMPA. After a one-year build-up phase and construction of a new lab in Buchs, Switzerland, a corporate competence centre for CRM production was established in 2006. A specially designed metrological weighing room with a series of state-ofthe-art balances represents the core of the new facility. As a positive confirmation of these efforts the Buchs CRM lab achieved double accreditation as a CRM producer according to ISO/IEC 17025 and also ISO Guide 34.[2] Today a series of CRMs are produced in the new lab demonstrating the declared intention of Sigma-Aldrich to become a key player not only in the analytical standards business but also in the field of production and distribution of CRMs.

\section{The Key Role of CRM in Chemical Metrology}

In metrological chemistry tremendous progress was made to improve the comparability of measurement results from laboratories of different nations. With more than 70 comparison studies on the highest metrological level (Key Comparisons) NMIs from all over the world demonstrated their analytical capabilities through analytical measurements of the very highest quality. ${ }^{[3]}$ In addition a lot of communication work was done through publications and presentations to familiarise the broader community with metrological concepts such as traceability and measurement uncertainty. Without question these activities of the NMIs are of highest importance since fundamental metrological concepts form the basis for any realisation of these concepts in practice. But even after many years of metrological awareness training not all these concepts are fully understood by the customers, i.e. testing laboratories. Very often users have difficulties to properly calculate uncertainty budgets for their measurement values: significant uncertainty contributions are overlooked or neglected many times. Traceability is often still understood as comparability to a NMI but not to SI using the certified value of a CRM. There are many reasons for this gap 
in understanding. One of the key issues is of course the fact that in chemical metrology there is no 'standard mole' as a single fix point to which all measurements can be compared. In chemistry traceability is mostly achieved through comparison of a measurement result to a well-defined reference, i.e. a CRM. In addition every chemical measurement result can be seen as one point in a three-dimensional space (representing the entirety of chemical measurements) which can be briefly defined by the three parameters analyte type, matrix composition and analyte concentration (Fig. 1). If one of these three parameters is changed the type of chemical measurement also changes significantly. In terms of traceability this means that in a perfect world an infinite number of CRMs should be available to cover the full three-dimensional space of all the chemical measurements. For every chemical measurement an appropriate CRM would be available to achieve traceability. Within the metrological community the question about comparability of measurement results is omnipresent and many times the question was asked: "how far does the light shine". ${ }^{[4]}$ Is the measurement of $200 \mu \mathrm{g} \cdot \mathrm{kg}^{-1}$ of lead in a wine matrix comparable to the measurement of $200 \mu \mathrm{g} \cdot \mathrm{kg}^{-1}$ of lead in a rice matrix (scenarios 1 and 2 in Fig. 1)? And if yes, to which extent in terms of measurement uncertainty? What happens in terms of comparability when not only the type of matrix is changed but also the concentration and the type of analyte (scenarios 1 to 4 in Fig. 1)? Of course a conclusive answer to this question cannot be given in this article. But it appears that a high number of available CRMs strongly supports the realisation of the traceability concept in practice. Only when many different CRMs are available to the end user can this three-dimensional space be populated with enough fix points so that every individual chemical measurement can be checked with known materials. Since every organisation underlies certain limitations in resources not all the required CRMs can be developed at NMIs alone. So it is essential that also industrial parties are active in the field of CRM development under compliance with ISO Guide 34 and ISO/ IEN 17025.

\section{Challenges in Industrial CRM Development}

When we talk about CRM development in an accredited, industrial environment this immediately brings up the question about the project profitability. It should not be discussed in this article whether it is good or bad when companies ask for financial justification before a new project is launched or not. It simply should be stated here as an axiomatic fact that industrial projects must generate some profit (at least in the long run) and therefore this is also the basic requirement for the new competence centre for CRM development at SigmaAldrich. When a new CRM is brought to the market it can only generate profit to the CRM producer when the customer is willing to buy it. And this is very often not only a matter of quality and documentation of a CRM but very often also a matter of price. Not only the CRM producer but also the end user, i.e. a testing laboratory, underlies financial pressure. Of course, all the testing labs following the ISO/IEN 17025 accreditation are encouraged to use CRMs if they are available. The use of a CRM is not only valuable for accredited parties but also for all the other laboratories without an accreditation. It is important that CRMs are not only available to the customer but also competitively priced. From a metrological point of view a CRM represents

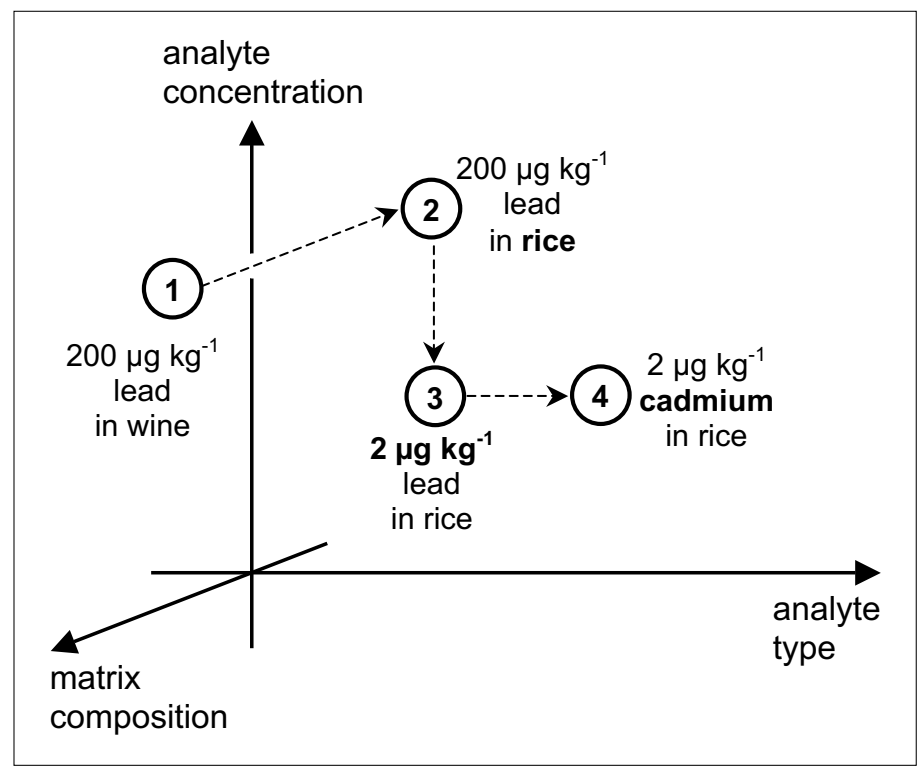

Fig. 1. The threedimensional space covering the entirety of all chemical measurements is defined through three axes: analyte type, matrix composition and analyte concentration. CRM represent the fix points in this space. the link from the metrological concept itself to its realisation in practice. And as a consequence every CRM which is available but not affordable does not bring any value either to the metrological community or to the end user. It will be an ongoing challenge for the industry to fulfil highest metrological requirements on the one hand (the 'as good as possible' approach) and to satisfy customers needs on the other hand (the 'fit for purpose' approach). In the next three sections of this article it will be demonstrated that this challenge can be met and that it is possible to produce and distribute CRMs of highest quality in an industrial environment.

\section{Comprehensive CRM Starting Material Characterisation}

\subsection{Realisation of Traceability for Inorganic CRM Development}

An appropriate way to achieve traceability to the SI system is by weighing known high-purity compounds. This follows from an almost unique feature of chemical measurement: $100 \%$ purity forms a natural reference value which cannot be exceeded. Coupled with widely available and excellent reference data for atomic weights of elements, ${ }^{[5]}$ and with additional data on physical parameters such as density, a high-purity material represents a local, practical realisation of concentration units through conversion of mass to molar quantity. Therefore, calibration with high-purity materials is accordingly a valid method of establishing traceability in analytical chemistry. ${ }^{[6]}$

With this traceability approach, a comprehensive characterisation of the starting material is of crucial importance. When, for example, a chloride reference is made from sodium chloride, the exact chloride content of the salt can be determined with very low uncertainty. However, determining the purity of a substance with a value of more than $99.95 \%$ requires an analytical method which gives results that have much less than $0.05 \%$ relative measurement uncertainty. This is practically impossible to achieve for most analytical techniques.

As a consequence, the common sense method for characterisation of high purity materials is the so-called impurity approach whereby as many impurities as possible are measured in a selected material by as many different analytical techniques as necessary. The sum of all impurities (and also the contribution from the potential impurities below their detection limit) is then subtracted from the maximum purity of $100 \%$. With this approach, it is possible to assign a reliable purity statement to high-purity materials, even for high-purity 


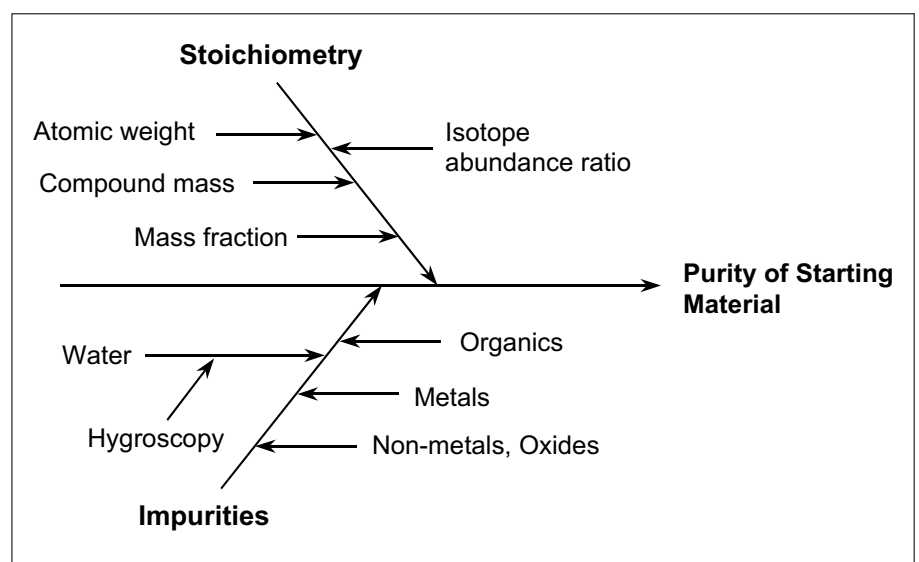

Fig. 2. Cause-effect diagram of relevant contributions to starting material purity.

grades ( $>99.99 \%)$. Of course, this approach gives reliable results only if much effort is put into the impurity investigations. For example, it makes no sense to look for the thirty most common metallic impurities in a high-purity sodium chloride, while at the same time overlooking the major impurities, bromide and water. How the various types of impurities contribute to the overall starting material purity is shown in the cause-effect diagram in Fig. 2.

\subsection{Starting Material Purity Declaration}

When considering high-purity materials, one should always look closely at the details, especially how the purity is defined. The following nomenclature has been established: When a material is assigned to be $>99.99 \%$ pure based on metallic impurities only, this is declared as an $\mathrm{m} 4 \mathrm{~N}$ material. Here, the ' $\mathrm{m}$ ' signifies that only metallic impurities are identified, and the ' $4 \mathrm{~N}$ ' stands for the 'four nines' $(99.99 \%)$ purity of the material. In some cases, such 'm-characterised' materials are also defined as 'metals base'. It is important to remember that the number of investigated elements is of great importance. All too often, certificates of analyses from industrial producers report only a very few number of trace metals.

A more reliable purity statement is provided when not only metallic impurities are identified, but also non-metals, anions, oxides and residual water. In this case a ' $\mathrm{t}$ ' (for total) replaces the ' $\mathrm{m}$ ' (for metals only). It is certainly no surprise that in many cases the reported purity (number of nines) is lower when the sum of all impurities, not only the metals, is reported. A material declared as a $99.9999 \%(\mathrm{~m} 6 \mathrm{~N})$ purity can be 'downgraded' to a $99.9 \%$ $(\mathrm{t} 3 \mathrm{~N})$ when also taking non-metallic impurities into account ( $c f$. Kipphardt, this issue). ${ }^{[7]}$ Of course, the total impurity approach requires more analytical effort and is more costly. On the other hand, the approach leads to greater reliability.

\subsection{Specific Aspects in High-Purity Salts and Metals Characterisation}

There are many challenges and also knock-out criteria during the process of evaluating and choosing appropriate starting materials.

\subsubsection{Residual Water}

When using inorganic compounds as starting material there can be water within the crystal structure coming from production and purification processes. When for example $\mathrm{NaCl}$ is used as starting material for chloride calibration solutions, drying at $110^{\circ} \mathrm{C}$ will remove adsorbed moisture but is not sufficient to ensure complete dryness of the salt. Investigations with TGA-MS show that only above $450{ }^{\circ} \mathrm{C}$ does $\mathrm{NaCl}$ completely lose its water. Since the amount of water can be more than $0.1 \%$, failure to remove it has a significant impact on the purity results. To guarantee complete dryness, $\mathrm{NaCl}$ has to be dried at $500{ }^{\circ} \mathrm{C}$ for at least four hours. ${ }^{[8]}$

\subsubsection{Stoichiometry}

$\mathrm{Na}_{2} \mathrm{HPO}_{4}$ is a good source as starting material for phosphate calibration solutions. During the evaluation of a particular batch of $\mathrm{Na}_{2} \mathrm{HPO}_{4}$, HR-ICP-MS and ICPOES (for metals), TGA-MS (for water), IC (for anions) and combustion analysis (for carbon) were used to measure purity. Even though 73 different impurities were identified, one significant impurity was missed. When a second IC method was run, significant levels of the dihydrogen analogue, $\mathrm{NaH}_{2} \mathrm{PO}_{4}$, were found in the starting material. This affected the total content of phosphate in the starting material due to the different stoichiometry of the impurity. Many compounds are non-stoichiometric to some degree and it is often a challenging task to prove that a particular material has the theoretical stoichiometry.

\subsubsection{Isotope Abundance Ratio and Atomic Weight}

Most elements, rather than being naturally monoisotopic, are composed of a number of stable isotopes with almost fixed isotopic abundance ratios. Since these ratios are not constant for a number of elements, it can lead to significant variations in the molecular weight for different compounds containing these elements. An important example is lithium where the IUPAC recommended atomic weight (also named mean relative atomic mass) $A_{r}(\mathrm{Li})$ is 6.941 and the mole fraction of ${ }^{7} \mathrm{Li}$ is 0.92409 (NIST L-SVEC lithium carbonate, $\mathrm{Li}_{2} \mathrm{CO}_{3}$ ). In materials of natural terrestrial origin the mole fraction of ${ }^{7} \mathrm{Li}$ can range from 0.9227 to 0.9278. ${ }^{[9]}$ When using $\mathrm{Li}_{2} \mathrm{CO}_{3}$ as a starting material for a lithium calibration solution, this can lead to a variation of the lithium content of up to $0.06 \%$ in the final solution. Additionally most commercially available lithium has been processed and is artificially depleted in the ${ }^{6} \mathrm{Li}$ isotope, which is of strategic importance for some countries. Boron is another element whose compounds can show deviations from the IUPAC natural isotope abundance ratio. First of all, there are small variations from natural isotope separation processes and secondly ${ }^{10} \mathrm{~B}$ has a very high neutron capture section that could be used in radiation hardened semiconductors, which makes it attractive to separate this isotope artificially from the natural isotopic mixture and leads to ${ }^{10} \mathrm{~B}$ depleted materials, that no longer show a mean atomic weight according to the IUPAC values.

\subsubsection{Non-metallic Impurities}

With pure metals as starting materials, it is usually necessary to take into account non-metallic impurities like $\mathrm{C}, \mathrm{H}, \mathrm{O}$ and $\mathrm{N}$. Oxygen, often at a few hundred $\mathrm{mg} \cdot \mathrm{kg}^{-1}$ but rarely exceeding $1000 \mathrm{mg} \cdot \mathrm{kg}^{-1}$, is found associated with many high-purity metals. In terms of the 'tXN' nomenclature, this means that a $\mathrm{t} 3 \mathrm{~N}$ purity $(>99.9 \%)$ is mostly not possible unless the starting material was produced under an inert, oxygen-free atmosphere. On the other hand, for certain types of metals the content of oxide impurities is not relevant. However, more often than not their existence must be considered. Since the quantitative determination of oxide impurities in metals is rather difficult, it may be acceptable to not account for the oxygen content itself, but to introduce an estimated contribution into the uncertainty budget of the purity calculation.

The problems outlined above taught us a few important lessons with regard to evaluating high-purity starting materials. ${ }^{[10]}$

i) Whenever possible and feasible, choose a pure metal over a compound as a starting material. Metals avoid problems with stoichiometry, residual water and hygroscopy. Also, because of their manufacturing process, metals normally do not contain appreciable amounts of non-metallic, anionic or 
organic impurities. Another advantage of using metals is that they can be weighed more easily than salts and powders and there is no risk of electrostatic charge up during the weighing manipulations. Even when the surface leaching and the dissolution of metals in acids take longer, using pure metals normally leads to lower uncertainties.

ii) If a salt must be used as a starting material, it is of utmost importance to eliminate all of the above-mentioned problems. Check for traces of residual water, control hygroscopy, know the stoichiometry and conduct comprehensive analysis of metallic, non-metallic and anionic impurities. With this information, it is possible to properly characterise the salt. However, for the above-mentioned reasons, high-purity compounds tend to have higher uncertainties than metals.

\section{Production of High-Precision Calibration Solutions}

\subsection{Pre-Treatment of Starting Materials}

The surface of metallic starting material is pre-cleaned by acid etching ( $c f$. Kipphardt this issue ${ }^{[7 b]}$ ). Diluted ultrapure nitric acid, hydrochloric acid or a mixture of nitric acid and hydrofluoric acid is used in most cases. The etched metal shots are transferred first into high-purity water through washing with water several times and afterwards transferred into acetone. Finally, the metal shots are dried under argon gas. These procedures are all performed in a clean room environment to avoid contamination with dust.

When salts are used as a starting material, they are normally dried in large, flatbottomed borosilicate glass or VYCOR dishes under specified drying conditions. The air supply to the high temperature oven is connected to a clean room bench. The dried salt is then brought to room temperature in a desiccator over anhydrous magnesium perchlorate.

\subsection{High-Precision Weighing}

High-precision balances are essential tools for mass measurements with low uncertainties. However, the environment around the balance as well as operator technique and know-how are also of critical importance. ${ }^{[11]}$

For vibration-free working the balances stand on three-point supported granite tables weighing up to $1000 \mathrm{~kg}$. Static electric charge-up of surfaces can be a source of uncontrolled gross errors in weighing. Hence aluminium rather than plastic vessels are used whenever possible. Such gross errors originating from static electric surface charges are not part of the model equation for the measurand and are not included in the uncertainty statement for the result. Gross errors need to be avoided in any case. For high-precision mass measurements a high-voltage static charge dissipater is especially useful when weighing dry non-conductive salts.

Temperature, humidity and air pressure in the weighing room need to be monitored since these data are necessary to calculate the air buoyancy bias. Air buoyancy bias has to be corrected since the balances are calibrated with reference weights having a density of approx. $8000 \mathrm{~kg} \cdot \mathrm{m}^{-3}$ while many starting materials have higher or lower densities. As a consequence, the weighing results are affected up to $0.1 \%$. Of course, all the weighing data are directly traceable to the SI unit $\mathrm{kg}$ by calibrating the balances with SI-traceable calibration weights. Only calibration weights fulfilling OIML class E2 and F1 are used to ensure highest quality of the weight measurements. Besides the normal calibration weights (density $8000 \mathrm{~kg} \cdot \mathrm{m}^{-3}$ ) calibration weights with a density of $2760 \mathrm{~kg} \cdot \mathrm{m}^{-3}$ (Anticorrodal) are also used to double-check the air buoyancy correction. All of the highprecision balances are periodically checked and calibrated by a third party and certified according to DKD guidelines

\subsection{Dissolving, Homogenisation and Bottling}

Every starting material is dissolved in high purity acid - mostly nitric or hydrochloric acid - following procedures specific for each material. This is important since the reaction behaviour of metals can differ greatly from one metal to another. Some of the metals are passivated immediately by surface oxidation when they come into contact with concentrated nitric acid and the dissolution reaction is stopped. For example, nickel can be dissolved overnight in diluted nitric acid at higher temperatures while other metals, such as magnesium, are much more reactive and dissolution in concentrated acid leads to uncontrolled and vigorous aerosol formation. A loss of starting material would be the negative consequence. Metals showing such a dissolution behaviour are normally dissolved very slowly in dilute acids followed by cooling for several hours. Obviously, all the dissolution reactions are done in totally inert bottles comprised of materials such as FEP or PFA.

After dissolution, the so-called stock solution containing the total amount of dissolved analyte is quantitatively transferred into the large PVDF mixing container. While the container is standing on the balance, the batch is filled with high purity water until the calculated total mass of the final solution is reached. This gravimetric approach allows a highly precise adjustment of the final concentration of the calibration solution. The solution is then homogenised by overhead tumbling of the PVDF container for several hours. With this technique it can be assured that the solution has no measurable inhomogeneity. This was demonstrated when this equipment was used for the preparation of samples for interlaboratory comparisons at the highest metrological stage. ${ }^{[12]}$

Last but not least, the standard solution is bottled under clean room conditions avoiding any contamination during the transfer process of the liquid. The bottles containing the CRM are then properly labelled, sealed into multilayered non-transmissible bags and stored under controlled conditions.

\subsection{Traceability Measurements}

In addition to the certified gravimetric value which is traceable to SI unit $\mathrm{kg}$, the starting material and the bottled solution are analysed by the most precise chemical analytical method available such as titrimetry or HP-ICP-OES. ${ }^{[13]}$ These direct measurements are done against internationally accepted certified reference materials and shall prove the absence of a significant bias during characterisation of the starting material and production process. Fig. 3 shows data from the direct titrimetric analysis of different starting material contents traceable to NIST Standard Reference Materi$\mathrm{als}^{\circledR}$ compared to the 'impurity approach'.

\section{Storage and Stability of CRM Solutions}

The ideal container for standard solutions is totally inert, will not adsorb analyte, does not leach impurities into the solution, is impermeable toward the solvent and atmosphere, and is easy to handle and store. It is likely that no container material will ever meet all of these requirements and still be affordable.

Some CRM producers use ampoules made of glass or of quartz for standard solutions. The use of ampoules offers the advantage of an absolute air-tight container. On the other hand it suffers from the impossibility to reseal the container. When an ampoule is opened the CRM must be consumed at once. This sometimes leads to more waste and/or one is forced to adapt working processes. Therefore many users prefer a container that can be easy handled and opened and closed as required. In terms of trace impurities the leach out from glass and to a lower extent from quartz into water-based solutions is significantly higher than from high-density polyethylene (HDPE) bottles.

Because of these reasons the HDPE bottle in combination with aluminium-coated bags is one of the most suitable materials available today. HDPE bottles fulfil the de- 


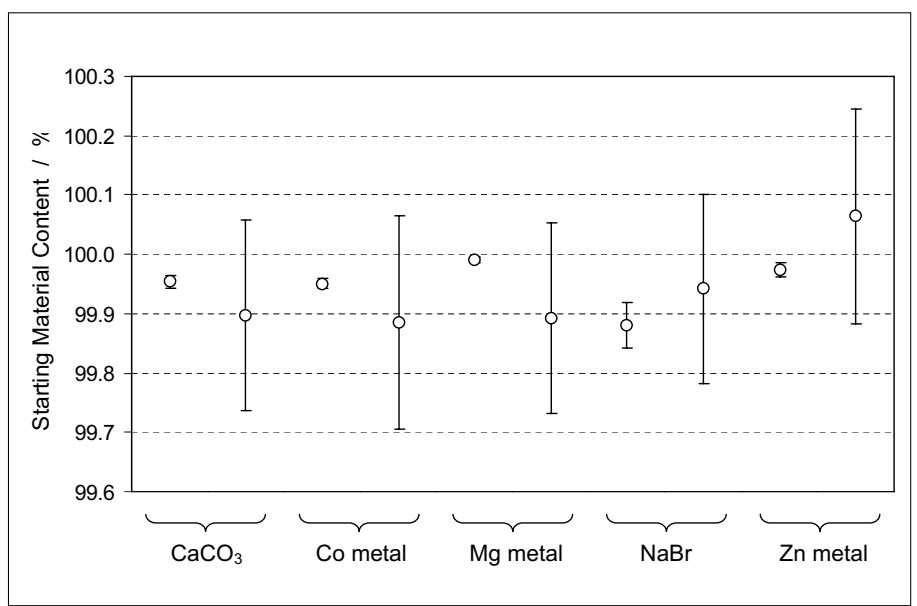

Fig. 3. The purities of starting materials are determined by calculation $100 \%$ minus all traces, analysed using ICP-MS, ICP-OES, AAS and IC (left values). The right values show the purities of the starting materials by direct titrimetric measurement (complexometry or argentometry) traceable to NIST SRM 728 and 999.

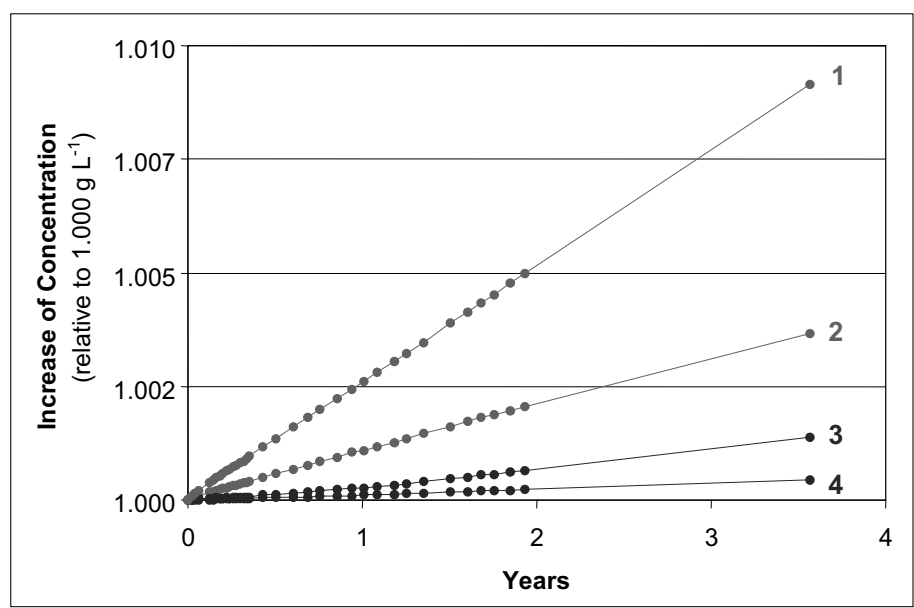

Fig. 4. Transpiration behaviour of aqueous calibration solutions in 100 $\mathrm{mL}$ HDPE bottles at different temperatures. $1: 30^{\circ} \mathrm{C} /$ without $\mathrm{Al}-\mathrm{bag} ; 2$ : $23^{\circ} \mathrm{C} /$ without Al-bag; $3: 30^{\circ} \mathrm{C} /$ in Al-bag; $4: 23^{\circ} \mathrm{C}$ in Al-bag. mand for the absence of trace contaminants; most elements cannot be detected even at $\mathrm{ng} \cdot \mathrm{L}^{-1}$ concentration levels when the bottles are leached with $2 \%$ nitric acid. Some omni present contaminations (e.g. calcium and sodium ions) might be found at very low $\mu \mathrm{g} \cdot \mathrm{L}^{-1}$ levels, which is not typically a problem for $1 \mathrm{~g} \cdot \mathrm{L}^{-1}$ standards. It should be kept in mind that the impurities found in the final calibration solution not only come from leaching out of the container but also from the starting material and the matrix (water, acid and/or base).

HDPE bottles are known to lose solvent through transpiration through the container wall. The rate and extent of loss depends on temperature, thickness of the wall, its shape and surface. Solvent is depleted more rapidly when the surface-to-volume ratio is high. Consequently, attention should be paid to storage conditions when small bottles are used. When HDPE bottles are welded into aluminium-coated bags the loss of solvent is reduced enormously. The increase of analyte concentration in HDPE bottles caused by solvent transpirations was investigated comprehensively during an EMPA study and is showed in Fig. 4.

There are several possibilities how to deal with the fact that even with a multilayered aluminium bag the analyte concentration in the HDPE bottle increases over time.

Every CRM bottle can have an individual 'temperature history' during storage at the warehouse of the CRM producer, during transport and in the customer's lab. For the correct analyte concentration in the CRM to be known one would need an equation for the change of analyte concentration depending on storage time and temperature. Users would also have a lower uncertainty at the beginning of the CRM validity than at the end. For convenient handling it is far too complicated to use an equation describing the analyte concentration depending on time and temperature with a variable uncertainty. This would certainly not be appreciated by the users.

A more conservative but straightforward approach is to include the possible solvent loss in the overall uncertainty. The maximum increase of analyte concentration at the highest specified temperature until the end of expiry (at $30^{\circ} \mathrm{C}$, four years) is taken as limit value. Assuming a rectangular distribution this value is divided by square root of three for the transformation into the standard uncertainty for storage condition. Following this procedure the uncertainty contribution coming from storage is a major part of the combined uncertainty of $0.2 \%$ which is guaranteed for the certified value over the entire shelf life of four years. ${ }^{[14]}$

\section{Conclusions}

Certified Reference Materials are of crucial importance for the realisation of metrological concepts in practice. Due to the nearly infinite number of different analytical measurements (defined by analyte type, analyte concentration and matrix composition) a huge number of commercially available CRMs is needed to ensure traceability of measurement results to internationally accepted references. Not only National Metrological Institutes but also industrial laboratories are playing an important role in the field of CRM production and certification. With the build-up of a double-accredited facility for CRM production at Sigma-Aldrich Switzerland it was demonstrated that highest metrological requirements are completely reconcilable with commercial aspects: with the launch of the new Trace CERT $^{\circledR}$ CRM portfolio Sigma-Aldrich underpins its declared intention to become a key players in the field of CRM production and distribution.

\section{Acknowledgement}

The authors kindly thank to Dr. Samuel Wunderli for fruitful discussions and his review work.

\section{Received: June 25, 2009}

[1] ISO/IEC 17025: 2005, 'General requirements for the competence of testing and calibration laboratories'.

[2] ISO Guide 34: 2000, 'General requirements for the competence of reference material producers'.

[3] BIPM Key Comparison Database: http://kcdb. bipm.org/.

[4] R. I. Wielgosz, Accred. Qual. Assur. 2001, 6, 329.

[5] M. E. Wieser, Pure Appl. Chem. 2006, 78, 2051.

[6] 'Traceability in Chemical Measurement', EURACHEM/CITAC Guide, 2003

[7] a) R. Matschat, M. Czerwensky, S. Pattberg, H.-J. Heinrich, Phys. Stat. Sol. 2002, 189, 107; b) H. Kipphardt, R. Matschat, U. Panne, Chimia 2009, 63, 637.

[8] EMPA / BAM Certificate No. U3-0203, SigmaAldrich Product No. 71387, Lot 094048/1, available at http://www.sigmaaldrich.com.

[9] T. B. Coplen, J. K. Böhlke, P. De Bièvre, T. Ding, N. E. Holden, J. A. Hopple, H. R. Krouse, A. Lamberty, H. S. Peiser, K. Révész, S. E. Rieder, K. J. R. Rosman, E. Roth, P. D. P. Taylor, R. D. Vocke, J. R. Xiao, Y. K. Xiao, Pure Appl. Chem. 2002, 74, 1987.

[10] J. R. Moody, R. R. Greenberg, K. W. Pratt, T. C. Rains, Anal. Chem. 1988, 60, 1203A.

[11] A. Reichmuth, S. Wunderli, M. Weber, V. R. Meyer, Microchim. Acta 2004, 148, 133.

[12] M. Weber, J. Wüthrich, S. Rezzonico, Microchim. Acta 2004, 146, 165.

[13] M. L. Salit, G. C. Turk, A. P. Lindstrom, T. A. Butler, C. M. Beck II, B. Norman, Anal. Chem. 2001, 73, 4821 .

[14] Trace CERT ${ }^{\circledR}$ - Traceable Certified Reference Materials. Part 1: Analytix, Vol. 5, 2006 and Part 2-5: Analytix, Vol. 1-4, 2007, available at www.sigmaaldrich.com/analytix. 